\title{
Extensive Percolation in Reasonable Idiotypic Networks
}

\author{
R.J. De Boer
}

Bioinformatics Group, University of Utrecht, Padualaan 8, NL-3584 CH Utrecht, The Netherlands

\section{Abstract}

We analyse a mathematical model specifying profound networks of idiotypic interactions amongst B cell clones. In the model we incorporate only fundamental assumplions about ldiotypic Interactions. Nevertheless, this "reasonable" idiotypic network model displays very "unreasonable" behaviour. As soon as the network is formulated as a profound network structure it displays "extensive percolation" of idiotypic signals. Thus the first antigenic perturbation of the network is expected to affect most of the clones. We have attempted to solve these problems by extending the model with seemingly important theoretical and/or immunological insights, but as yet we have falled. Therefore, our present interpretation of these "unrealistic" results is that immune systems do not function by means of profound network structures.

\section{Introduction}

Jerne's [1] idea that immune systems are regulated by networks of idlotypic interactions has roused considerable interest among both theoreticlans and experimentalists. Immune systems perform complex functions such as specific immune reactions, the specific memory phenomenon, and self non-self discrimination. Although it remains an open question, it is concelvable that immune systems perform these tasks by means of these proposed network properties. To this end, immune systems have often been compared to neural networks [1-3]. Neural networks, for which Hopfleld equations provide the general paradigm system, are capable of complex computational processes (such as learning and memory) [4]. The similarity between neural and Idiotypic networks, and the powerful computational properlies of neural networks, have probably shaped our intultion: in immunology theoreticians are generally inclined to think that idiotyplc Interactions do indeed play an important role in immunoregulation.

Because immunoregulation by means of these network properties is little more than an assumption, we here analyse it by means of a "bottom up" approach. Starting with basic assumptlons about Idiotypic Interactions, and their effects on B cell prollferation, we develop a "fundamental" Idlotypic network model. Such a model might eventually become comparable to neural network models, l.e. might eventually obtain comparable (immuno)computational properties. We thus investlgate whether simple idiotyplc interactions amongst B cell clones can Indeed account for the proposed regulatory network properties. Our previous analysis [5-7] gave an ambiguous answer to this 
question. On the one hand, it was shown that our idiotypic networks could not account for the control of proliferation. Proliferating clones in the network suppress their lidiotyplc partners long before these become sufficiently suppressive to control the proliferation. On the other hand, It was demonstrated that idiotypic interactions can easily account for memory phenomena: the interaction between an idlotypic and an anti-idiotypic clone brings about three different equilibria, one of which corresponds to an immune state for the idiotypic clone. Thus idiotypic network theory accounted for immunological memory, but could not account for proliferation regulation (i.e. for supprassion) [6]. We will here test the robustness of these memory phenomena by analysing profound networks consisting of many (i.e. 100) instead of just a few (i.e. 2) clones.

\section{The assumptions}

We here consider clones of B-lymphocytes. We assume that the B-lymphocyte populations are regulated by three processes: 1) influx of newborn cells from the bone marrow, 2) normal turnover of cells, and 3) proliferation. It is further assumed that idiotypic interactions influence the rate of cell proliferation. The network consists of $\mathrm{N}$ clones, each of which is identified by a unique random receptor. A clone $\left(X_{i}\right)$ recognises another clone $\left(X_{j}\right)$ if (part of their respective receptors can be matched complementarily. The accuracy of this match specifies the affinity ( $\left.A_{i i}\right)$ of the idiotypic interaction between $X_{i}$ and $X_{j}\left(0 \leq A_{i j} \leq 1\right)$. Each clone can also be stimulated by its antigen $(\mathrm{Ag})$.

Influx/efflux. In the absence of idiotypic interactions the clone size is delermined solely by the balance between the source $\left(S_{i}\right)$ of cells from bone marrow and the death (D) of cells in the periphery. This suffices for a stable virgin state at a clone size of S/D. Because the influx of cells per clone is small, and the rate of cell lurnover is high, virgin populations are typically small. In the virgin state idiotypic interactions are therelore weak or absent.

Symmetry. If idiotypic recognition is based on complementary matching and receptor crosslinking. idiotypic interactions are necessarily symmetric. If idiolype "i" matches "j", "j" should also match "I"; If "I" antibodies crosslink "j" receptors, "j" antibodies can do the same. Hoffmann [8,9] first proposed this simple and attractive symmetry theory; he [9] and Jerne [10] review empirical data that support symmetry theory (Jerne's "preferred partners"). Clones never recognise themselves (1.e. we set all $A_{i l}$ to zero).

Dose response relation. Because B cells are activated by the crosslinking of the antigen receptors, it is to be expected that the rate of cell activation will increase if the concentration of the crosslinking agent (here antigen or antl-Idlotypic antibody) increases. However, when these concentrations become too high the efficacy of cell activation by receptor crosslinking decreases [11]. Thus, the present model is based on the elficacy of cell activation and proliferation induction (by receptor crosslinking); inhibitory interactions are incorporated as the reduction of the proliferation rate. This argument corresponds to a bell-shaped proliferation dose response curve, l.e. the kind that can be found in any immunology textbook. 
The model. The rate of cell proliferation is governed by a growth dose response function $G\left(X_{1}, A g \mid\right.$, $\alpha\left(d_{1}\right)$, which depends on 1) the size of the clone (a buffering term), 2) the antigen, and 3) the total amount of anti-idiotype $\left(\alpha \mid d_{i}\right)$. The function $G\left(X_{1}, A_{g_{1}}, \alpha \mid d_{1}\right)$ is maximally one; prollferation per cell then proceeds at a rate $P$ per day. The function (eq. 2) is a log bell-shaped curve: antl-idiotype concentrations can become too high. This reduces the crosslinking and hence the rate of cell proliferation. For reasons of simplicity, antigen can only increase (up to a certain maximum) the rate of cell prollferation: the antigen dose response curve is a simple saturation function. Antigen $\left(A g_{i}\right)$ cannot grow and is either removed by the clone that recognises $i t\left(X_{1}\right)$, or is incorporated as a constant $(K=0)$. We thus propose the following model:

$$
\begin{aligned}
& \alpha / d_{i}=\sum_{j=1}^{N} A_{i j} X_{j} \\
& G\left(X_{i}, A g_{1}, \alpha \mid d_{1}\right)=\frac{A g_{i}+\alpha l d_{1}}{P_{1}+F 0 X_{1}+A g_{i}+\alpha \mid d_{i}} \cdot \frac{P_{2}}{P_{2}+\alpha l d_{1}} \\
& x_{i}=S_{1}-D \circ X_{1}+P_{0} X_{j} \circ G\left(X_{1}, A g_{1}, \alpha \mid d_{1}\right) \\
& A g i=-\frac{K_{0} A g_{10} X_{1}}{K_{1}+X_{1}}
\end{aligned}
$$

Parameters. The parameter selting is: $S=10$ cells $d^{-1}, D=1 d^{-1}, P=1.5 d^{-1}, P_{1}=10^{3}, P_{2}=10^{6}$, $F=0.01, K=1, K_{1}=10^{5}$. Buffering ensures that large $X_{1}$ populations $\left(F_{\circ} X_{1}=P_{1}\right)$ cannot be stimulated by small antigen and/or anti-idiotype concentrations [6]. The virgin population density equals $S / D=10$ cells. The influx is sllghily different for each clone (to prevent settlement into unstable equilibria): $S$ has a mean of 10 cells per day with a $10 \%$ standard deviation. Virgin populations are too small to evoke proliferation $\left(S / D \ll P_{1}\right)$ : idiotypic interactions are negligible in the virgin state. Maximum proliferation proceeds at a rate of P-D $=0.5$ cells per cell per day (this corresponds to a doubling time of about 16 hours).

Methods. The affinity matrix is either based on a complementary matching algorithm, or is drawn randomly from a uniform distribution between zero and one. The complementary matching algorithm resembles that of Farmer et al. [12] and Perelson [13]. Ours matches patterns (landscapes) defined by an array of real numbers (that are uniformly distributed between zero and one). The algorithm is described elsewhere [7]. The low-D models have been analysed by GRIND [14] which performs numerical 0-Isocline analysis and numerical integration by means of ROW4A [15]: High-D models are integrated by means of a variable step size Runge-Kulta-Merson integrator Implemented in NAG [16]. 


\section{Results and Discussion}

Memory. We now summarise our previous analysis of Immunological memory [6]. Consider a network of two clones: $X_{1}$ and $X_{2}$ that see each other with maximum affinity (l.e. $A_{12}=1$ ). This network will be stimulated with the antigen that is recognised by $X_{1}$ (i.e. $A g_{1}$ ). In Flg.1a this 2-D network is analysed statically: the curved lines are the $X_{1}^{\prime}=0$ and the $X_{2}=0$ isoclines. The region in which $X_{1}$ expands is shaded; $X_{1}$ proliferates in the large shaded region situated at an intermediate $X_{2}$ population size. The isoclines intersect in three stable equilibria: the virgin state (V) and two immune states $\left(I_{1}\right.$ and $\left.I_{2}\right)$. In the absence of antigen the system always remains in the virgin state; here idiotypic interactions are negligible. Fig. 1b shows the same picture with a trajectory (l.e. the fat line) that was initiated by the introduction of antigen $\left(A g_{1}=10^{4}\right)$. In response to antigen, $X_{1}$ proliferates (the trajectory moves to the right) which, in turn, evokes the proliferation of $X_{2}$ (the trajectory moves upwards). As a consequence of the proliferation of $X_{1}$, antigen is rejected (not shown) and the system settles into the $I_{1}$ equilibrium. In this new state the system is immune to $\mathrm{Ag}_{1}$ : reintroduction of $A g_{1}$ leads to rapid rejection because $X_{1}$ is already enlarged. Conversely, $X_{2}$ is suppressed in the $I_{1}$ state: introduction of $\mathrm{Ag}_{2}$ into this state never leads to rejection because $X_{2}$ fails to proliferate. Thus the previous exposure to $\mathrm{Ag}_{1}$ is specifically remembered by the network.

In both immune states both clones are enlarged, i.e. both clones proliferate. Thus the network maintains its immunity to $A g_{1}$ by the mutual stimulation between $X_{1}$ and $X_{2}$. The reason why $X_{2}$ is nevertheless said to be "suppressed" in the $I_{1}$ state is that it cannot proliferate any further. Any increase in $X_{1}$ decreases the rate of $X_{2}$ proliferation, whereas increasing $X_{2}$ increases $X_{1}$ proliferation. Antigenic stimulation of $X_{2}$ also fails to evoke additional proliferation and antigen cannol be rejected: $X_{2}$ is suppressed.
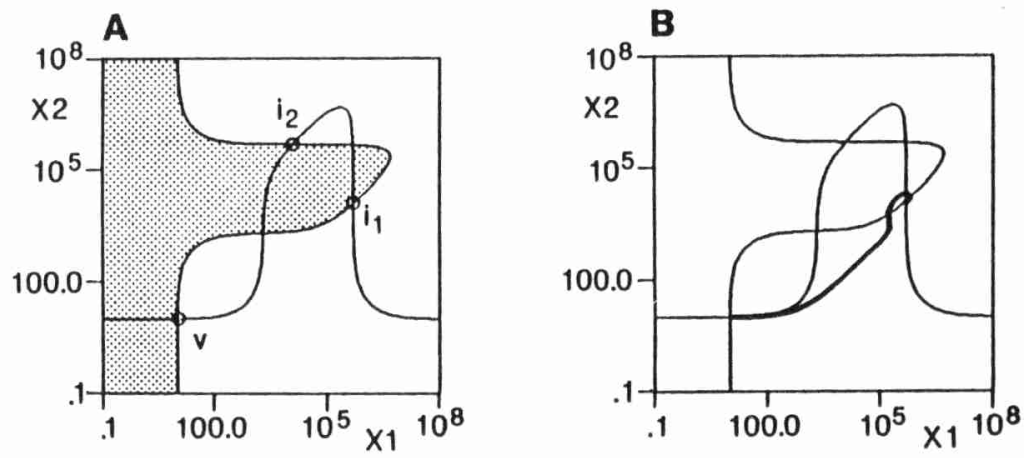

Figure 1. A 2-D network with maximum affinity $\left(A_{12}=1\right)$. Fig. $2 a$ : the $X_{1}^{\prime}=0$ and $X_{2}^{\prime}=0$ isoclines define 3 stable equilibria: a virgin state $(V)$ and two immune states $\left(I_{1}\right.$ and $\left.I_{2}\right)$. The $X_{1}>0$ region is shaded. Fig. 2b: the trajectory of a switch from the virgin state to an immune state $\left(I_{1}\right)$ as it is evoked by an antigen dose of $\mathrm{Ag}_{1}=10^{4}$ cells. 
Absence of fading. Now consider a system with a third clone $\left(X_{3}\right)$ that interacts with $X_{2}$. We again introduce $\mathrm{Ag}_{1}$ and, as a consequence, $X_{1}$ and $X_{2}$ switch to the $I_{1}$ equilibrium. In the $I_{1}$ equilibrium the anti-idiotypic $X_{2}$ population maintains the proliferation of the large idiotyplc $X_{1}$ population (note that $X_{1} \gg X_{2}$ ). It is therelore to be expected that $X_{2}$ should also be able to iniliale the proliferation of the (small, l.e. virgin) $X_{3}$ population. In our model we have implicitly (and quite reasonably) assumed that if should be easier to activate all cells of a small clone than of a large clone. Thus, on reasonable grounds, we expect $x_{2}$ to initiate the prollferation of $x_{3} . x_{3}$ as a consequence switches to an immune state that is comparable to that of $x_{1}$ in the $l_{1}$ state (not shown). This argument can however be continued: $X_{3}$ is expected to Initiate the proliferation of the clone(s) to which it is connected, and so on. We conclude that the idiotypic activation signal falls to fade during its propagation into the network: along the propagation pathways clones keep on switching to "immune" or "suppressed" states comparable to those described above. Elsewhere [7] we provide more evidence for this argument.

Connectivity thresholds. The percolation of the idiotypic signal through the network depends not only on its extinction rate (i.e. on the above-mentioned fading), but also on the connectlvity of the network. In highly connected networks the propagation pathway will branch several times, and the signal may fade away on one branch but proceed via another. We can analyse the connectwity properties of an idiotypic network by means of graph theory. A randomly connected, symmetric, idiotypic network corresponds to a (isotrophic) random undirected graph in which the idiotypic interactions are the edges (E) and in which the clones correspond to nodes $(N)$; see also Perelson [13]. The connectivity properties of isotrophic random undirected graphs (In which $E$ edges connect $N$ nodes equiprobably) were analysed by Erdos \& Renyl [17,18], in Kauffman [19]. Several results of random infinite graphs [17-19] are of interest for our (finite) random idiotypic networks. If E<<N only small isolated structures are found, In which any node is connected to a few others. Whenever the ratio of E/N exceeds 1/2, a threshold is reached and most nodes are interconnected in one enormous structure. As E/N increases still further, more isolated nodes are incorporated into this very large connected structure. (For finite graphs these thresholds soften to sigmolds). Note that in our ldiotypic networks, an average of one edge per two clones $(E / N=1 / 2)$ corresponds to an average of one idlotypic connection per clone.

The Erdos \& Renyl theory thus predicts that once the connectivity exceeds one Idlotyplc connection per clone, most clones suddenly become interconnected. Such connectivity however is extremely weak: If the connectivity of the ldiolypic network were to be still weaker, a number of clones would not be connected to the network at all. They would hence require other immunoregulatory mechanisms. Moreover empirical data suggest that one clone can be connected to very many (e.0. 40) clones [20-22]. It thus seems sale to assume that the network connectivity usually exceeds one connection per clone. According to the Erdos \& Renyl theory such an assumption however means that most clones are interconnected in one large structure. Moreover, If we combine this conclusiom with our "absence of fading" results, It follows that each perturbation of the network (by e.g. antigen) eventually affects all the clones. This is very unreasonable: any antigenic stimulation of 
the Immune system eventually forces almost all clones of the idiotypic network to switch to the "immune" or "suppressed" state. This unreasonable result, l.e. extensive percolation, however seems to be based on very reasonable assumptions and theories. At present we can see three possible explanations for this: 1) the Erdos \& Renyi theory is not applicable to biotic idiotyplc network structures, 2) we have omitted an essential element of the idiotypic network from our models, and/or 3) Immune systems do not function as an idiolypic network.

1. Random vs. Paltern networks. The Erdos \& Renyl theory is based upon random graphs. The connections In an idiotypic network are however not necessarlly random. We here investlgate the Impact of the presupposed randomness on the percolation results. We compare networks based on random connections of a random strength $\left(0 \leq A_{i j} \leq 1\right)$ with networks based on our complementary matching algorithm. The algorithm matches idiolypes that were drawn randomly; the resulting affinities are also scaled between 0 and 1. We refer to them as the "random" and the "pattern" networks respectlvely. In both networks the connectivity is measured by the average number of Interactions per clone (nc). In "pattern" networks the connectlvity is increased by increasing the size of the pattern (i.e. by allowing for more idiotopes per idiotype). We thus simulate networks of 100 clones; we perturb the network with antigen at day 100 (I.e. when It has attained the virgin state); antigen is removed after 25 days.

Two typical examples (of "pattern" networks) are shown in Fig. 2: one is located around the Erdos \& Reyni threshold (nc=1.04, Fig. 2a) and the other slightly above it (nc=1.36, Flg. 2b). This slight difference in connectivity accounts for an enormous difference in behaviour: in Fig. $2 \mathrm{a}$ the immune reaction involves 3 clones whereas that of Fig. $2 \mathrm{~b}$ involves 34 clones. This is mainly due to the

A

$\log 10$ Pop.

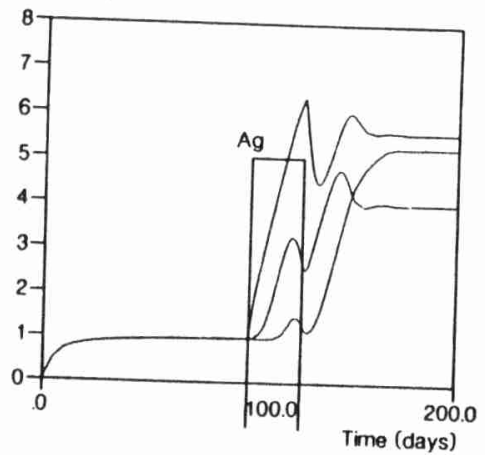

8

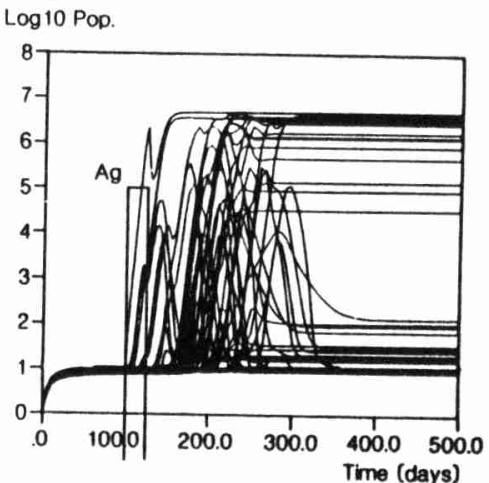

Figure 2. Examples of the behaviour of 100-D "pattern" networks. A network with on average 1.04 connections per clone (2a), and (2b) a network with 1.36 connections per clone. This is just around and just above the Erdos \& Reyni threshold. The Flgure only depicts the clones that are actually connected to the network stimulated by antigen (all other clones remain virgin). 
connectlvity properties: the clone that responded to the antigen was actually connected to 3 clones in $\mathrm{Fig}$. 2a and to 55 clones in Fig. 2b. In Fig. 3 we score similar network properties as a function of the connectivity of the network. We score 1) the "effective" dimension of the network, I.e. the number of clones that are actually connected to the clone stimulated by antigen. And, in the equillbrium that is finally reached, we score 2) the number of clones that are actually affected, I.e. that have left the virgin state, and 3 ) the depth of signal propagation, 1.e. the maximum idiotypic level affected. The first score measures the Erdos \& Reyni property; the other two link this with "absence of fading".

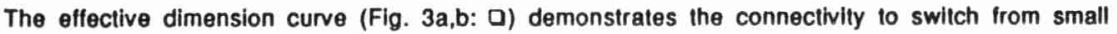
isolated structures to one large interconnected network around one connection per clone. The threshold is relatively smooth, but for the almost infinite ( $10^{7}$ clones) biotic immune system it is expected to be very steep. The number of clones that are actually connected to the clone stimulated by antigen (1.e. the effective dimension) hardly differ for "random" and "pattern" networks (Fig. 3a vs. 3b). We conclude that the Erdos \& Reyni results are applicable to our idiotypic networks which are based on the very reasonable complementary matching assumption.
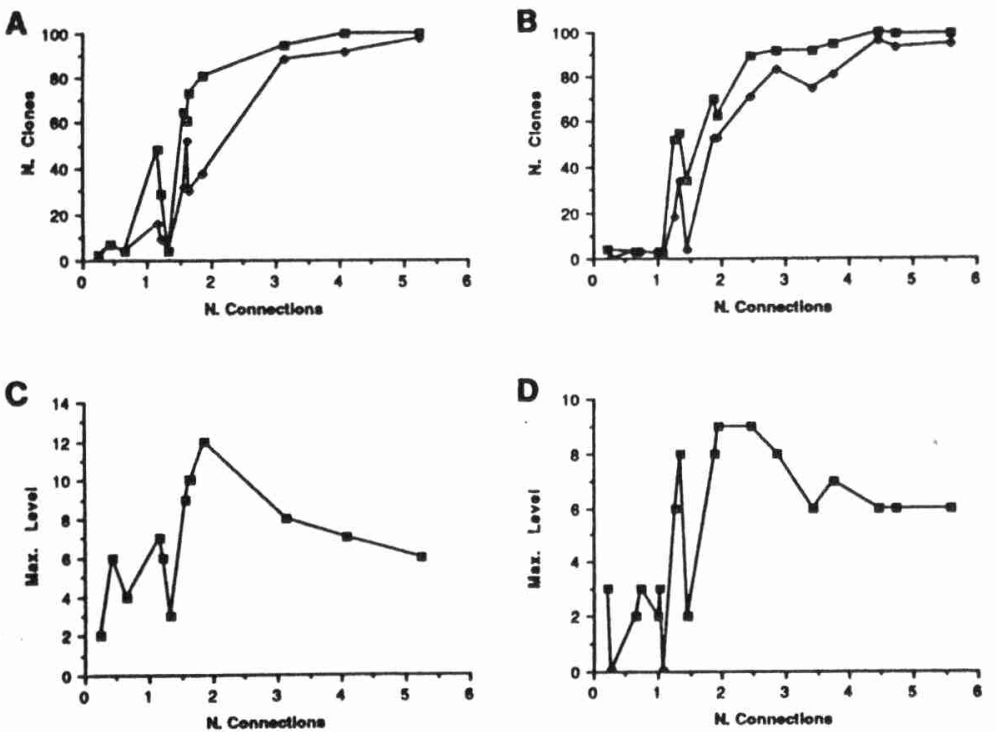

Flgure 3. The network properties of 100-D "random" (3a,c) and "patterm" (3b,d) networks as a function of the average number of connections per clone. Flg. 3a,b: the actual number of clones connected to the clone stimulated by antigen (D), and ( ) the actual number of clones that become affected by the signal, l.e. that leave the virgin state. Fig. 3c,d: the maximum idiotypic level reached by the lidiotypic signal. 
The two other properties that we score are also very similar lor "random" and "pattern" networks. The number of affected clones (Fig. 3a,b: 0) simply follows the effective dimension curve: a limited number of clones that are actually connected to the perturbed sub-network remain in the virgin state. Networks in which most clones have been affected by a previous perturbation are highly unresponsive to novel perturbations (i.e. to other antigens): antigens are either directly rejected because "their" clone is already immune or cannot be rejected because "their" clone is suppressed. Moreover, the percentage of suppressed clones increases if the network connectivity increases [5]. The maximum idiotypic level that is actually reached by the signal has a peak around 2-3 connections per clone. The maximum is about 10-12, but in 200-D systems we found higher values (e.g. 17). It takes the idiotypic signal several months to attain such a high idiotypic level (see Fig. 2b). During these months the network oscillates heavily, l.e. seems chaotic; we think such extenstve oscillations are unrealistic.

2. What is missing? The fact that we find unrealistic results with simple networks that incorporate only "realistic" assumptions might suggest that "apparently" we have omitted an essential element of the idiotypic network. We here investigate this possibility. Note that if we indeed were to find a process that solved our percolation problem this would pinpoint this process as playing an essential role in the control of percolation. This would be a very important result since, so far, idiotyplc network theory apparently lacks such a regulatory control structure.

Long-range inhibition. Segel \& Perelson [23] and Segel [this volume] develop an idiotypic network model that incorporates "long-range inhibition" and "short-range activation"; this combination is known to promote pattern formation. In our model, activation and inhibition are governed by the same affinity matrix, l.e. both interactions are of an identical range. We have, however, tested the "long-range inhibition" idea in our "pattern" networks [7]. We made suppressive interactions more general by requiring a shorter complementary match (i.e. a smaller idiotope) for suppression than for activation. This can even be defended on immunological grounds: It is concelvaible that anti-idiotypic antibodies that fail to crosslink idiotypic receptors might have sufficient affinity to these receptors to reduce (e.g. by temporary binding) the crosslinking process of another (high affinity) antibody. However, in our symmetric networks "long-range inhibition" falls to reduce the percolation. We find that the networks 1) can only slowly settle into equilibria, 2) seem more chaotic, and 3) still display extensive percolation. These counter-intuitive results are probably due to the fact that our networks do not discriminate between "activators" and "inhibitors". Whether a clone activates or inhiblts depends on the idiotypic circumstances. Thus "long-range inhlibition" can, after a long period of rather constant circumstances, Inhibit a clone from inhiblting. As a consequence of these changing circumstances that clone not only stops inhibiting but may even start to activate its idiolypic partners. Thus "long-range inhibition" seems to imply "long-range activation".

Antibodies. In our simple model we have lumped into one population $(X)$ B-lymphocytes and the antibodies that these cells produce. The elimination of antigen and the idiolypic comminication 
between clones (i.e. the transfer of the idiotypic signal) in fact occur via antibody molecules. Additionally, cells only differentiate into antibody producing plasma cells after they have proliferated [24]; this accounts for a delay in the idiotypic communication. Moreover, antibodies have a longer life span than cells [10]. Elsewhere [7], we have incorporated these additional features in our ldiotypic network model. Inslead of solving our percolation problems, the addition of these features generated new unrealistic results. The network no longer switches from virgin to immune states (that still exist), but, following the introduction and elimination of antigen, oscillates heavily from the $I_{1}$ to the $I_{2}$ state, and vice versa. This cycle never ends. It is sustained by the slow decay of the antibodies: high, l.e. suppressive, concentrations of antibody decay slowly because they have suppressed the network. This decrease in the antibody concentration however changes the idiotypic circumstances, l.e. the antibody concentration becomes stimulatory again. Thus cells initiate proliferation and start to produce new antibodies until the antibody concentrations again become suppressive. We have also incorporated the formation of complexes of idiotypic and anti-idiotypic antibody molecules. This failed to solve the percolation problem; moreover the above-mentioned cycle persisted.

$T$ cells. Idiotypic network theory was originally presented in terms of receptor interactions between B cells [1]; our model also considers B cells. T cells however play a crucial role in Immunoregulation. Idiotypic network theory can be extended with T cells in two ways. First, B cell proliferation and antibody production usually result from B-T cell cooperation. B cells present antigen (and/or idlotype) to the helper $T$ cells that in turn produce lymphokines. Thus idiotypic Interactions amongst $B$ cells require the concomitant activation of helper $T$ cells (in our model we have in fact assumed that all B cells receive sufficient help). We are at present analysing the conditions for percolation in a network model with helper T cells [De Boer \& Hogeweg, in prep.]. These preliminary results suggest that helper $T$ cells hinder the development of ldiotypic B-B cell interactions. Secondly, T cells might also form networks of idiotypic T-T cell interactions. Recent experimental data $[25,26]$ strongly suggest that such interactions play an important role in immunoregulation. Several complications however hamper the development of reasonable models of such T cell networks: 1) are T-T interactions MHC restricted and, as a consequence, do these $T$ cells express class II MHC which seems necessary for helper T cell interactions, and 2) do we have to Incorporate a separate class of (the very controversial) suppressor $T$ cells, or (which seems more Interesting) can the suppressor phenotype be the result of the idiotypic interaction? Untortunately, due to these open questions, these experimental data allow for the development of very many, seemingly equivalent, models. As yet, we conclude that the role that $T$ cells play in the percolation of Idiotypic B-B, B-T, or T-T signals remains to be established.

3. No functional network. The most interesting interpretation of our extenslve percolation problem is that immune systems apparently do not function by means of such profound network structures. The existence of functional idiotypic interactions amongst "classical" B cells was also questioned by Eichmann [this volume]. Additionally, Urbain [27; this volume] proposed his "broken mirror hypothesis" which implies that idiotypic interactions occur in pairs and not in the form of profound 
networks. If Idlotypic interactions were indeed to fail to form profound networks, the comparison with the powerful neural networks would break down, and we should no longer speak of immunoregulation by network properties. Even Jerne [10] speaks of "prelerred partners" In idiotyplc interactions. Also Cohn [28] questions the functional significance of ldiotypic networks. Thus it seems quite reasonable to conclude that our percolation problem is a consequence of our erroneous assumption that immune systems function by means of profound network structures. Our preliminary results which show that helper $T$ cells may hinder the development of functional idiotypic interactions would, if indeed immune systems do nol function by means of prolound network structures, provide a means of escaping from the seemingly inescapable ldiotypic network theory [De Boer \& Hogeweg, in prep.].

IgM networks. In empirical Immunology, however, idiolypic network theory has roused considerable interest recently. Several levels of specific idiotypic interactions amongst monocional antlbodies, i.e. "paper networks", have been described [29]. Moreover, it has been demonstrated that, in contrast to the adult immune system, B cells early in ontogeny have numerous idiotypic interactions $[21,22]$. Additionally, it has been suggested that the B cells from the early repertoire form a separate lineage of cells [30]. These lgM producing cell types most probably function independently of helper $T$ cells and are hence indeed expected to form prolound network structures [De Boer \& Hogeweg, in prep.]. It is thus concelvable that, early in ontogeny, Immune systems do form functional networks, and that these network properties disappear when the system matures. The possible significance, for e.g. self non-self discrimination, of such "early repertolre" networks seems an interesting point for further study.

Classical Immune systems. For the classical, l.e. adult, Immune systems that in fact display the phenomena of specific immune reactions, immunity, and tolerance, we apparently have to look for alternative modes of immunoregulation. One which remalns close 10 idlotyplc network theory is regulation by anti-idlotyplc suppressor $T$ cells $[25,26]$. Another, which seems more simpln (and less controversial) is the clonal helper $T$ cell response. The helper $T$ cell proliferation process has, due to autocatalytic IL2 production, proliferation thresholds [31-33] that account for several tolerance phenomena, including self non-self discrimination. Finally, antigen presentation by macrophages and/or subsequently by B cells might play an Important role in immunoregulation. We conclude that the clonal level of the immune system seems to provide sufficient Immunoregilatory processes to account for complex "computation" or "Information processing" .

\section{Acknowledgements}

This work has been done in close collaboration with Dr. P. Hogeweg, head of our Bioinformatics Group. I am grateful to Ms. S.M. McNab for linguistic advice. 


\section{References}

1. N.K. Jerne: Ann. Immunol. (Inst. Pasteur) 125C, 373-389 (1974)

2. G.W. Hoffmann: J. Theor. Biol. 122, 33-67 (1986)

3. F.J. Varela, A. Coutinho, B. Dupire, N.N. Vaz: In Theorelical Immunolegy, ed. by A.S. Perelson, Part Two, SFI Studies in the Science of Complexity, Vol. III (Addison-Wesley, 1988) pp. 359-375

4. J.J. Hopfield, D.W. Tank: Science 233, 625-633 (1986)

5. R.J. De Boer: In Iheoretical Immunelegy, ed. by A.S. Perelson, Part Two, SFI Studies in the Science of Complexity, Vol. III (Addison-Wesley, 1988) pp. 265-289

6. R.J. De Boer, P. Hogeweg: Bull. Math. Biol. (in press)

7. R.J. De Boer, P. Hogeweg: Unreasonable Implications of Reasonable Idiotypic Network Assumptions (submitted)

8. G.W. Hoffmann: In Lecture Notes in Blomathematics, ed. by C. Bruni, G. Doria, G. Koch, R. Strom, Vol. 32 (Springer, Berlin, 1979) pp. 239-257

9. G.W. Hoffmann: In Centemp. Tepics_ Immunoblole, ed. by N.L. Warner (Ed.), Vol.11 (Plenum Press, New York, 1980) pp. 185-226

10. N.K. Jerne: Immunol. Rev. 29, 5-24 (1984)

11. B. Goldstein: In Theoretical Immuneleax ed. by A.S. Perelson, Part One, SFI Studies in the Science of Complexity, Vol. III (Addison-Wesley, 1988) pp. 3-40

12. J.D. Farmer, N.H. Packard, A.S. Perelson: Physica 22D, 187-204 (1986)

13. A.S. Perelson: In Theoretical Immuneleax, ed. by A.S. Perelson, Part Two, SFI Studies in the Science of Complexity, Vol. III (Addison-Wesley, 1988) pp. 377-401

14. GRIND: Great Interater Differential Equations. R.J. De Boer, Bloinformatics Group, University of Utrecht, The Netherlands (1983)

15. B.A. Gottwald, G. Wanner: Computing 26, 355-360 (1981)

16. NAG: Numerical Algorithms Group. Mayfield House, 256 Banbury Road, Oxford OX2 7DE, United KIngdom (1984)

17. P. Erdos, A. Renyi: On the randem araphs 1, vol. 6. (Debrecar, Inst. Math. Univ. DeBreceniens, Hungary 1959)

18. P. Erdos, A. Renyl: On the randem araphs, Publ. No. 5, Math. Inst. Hung. Acad. Scl. (1960)

19. S.A. Kauffman: J. Theor. Biol. 119, 1-24 (1986)

20. J. Novotny, M. Handschumacher, R.E. Bruccoleri: Immunol. Today \& 26-31 (1987)

21. D. Holmberg, S. Forsgen, F. Ivars, A. Coutinho: Eur. J. Immunol. 14, 435-441 (1984)

22. D. Holmberg, G. Wennerstrom, L. Andrade. A. Coutinho: Eur. J. Immunol. 16, 82-87 (1986)

23. L.A. Segel, A.S. Perelson: In Theoretical Immunolegy, ed. by A.S. Perelson, Part Two, SFI Studies In the Science of Complexity, Vol. III (Addison-Wesley, 1988) pp. 321-343

24. F. Melchers, J. Anderson: Ann. Rev. Immunol. 4. 13-36 (1986)

25. O. Uider, T. Reshef, E. Beraud, A. Ben-Nun, I.R. Cohen: Science 239, 181-183 (1988)

26. D. Sun, D., Y. Qin, J. Chluba, J.T. Epplen, H. Wekerle: Nature 332, 843-845 (1988)

27. J. Urbain: Ann. Immunol. (Inst. Pasteur) 137C, 57.64 (1986)

28. M. Cohn: Ann. Immunol. (Inst. Pasteur) 137C, 64-76 (1986) 
29. M. Vakil, J.F. Kearny: Eur. J. Immunol. 16, 1151-1158 (1986)

30. K. Hayakawa, R. Hardy, M. Honda, L.A. Herzenberg, A.D. Steinberg, L.A. Herzenberg: Proc. Natl. Acad. Scl. USA 81, 2494-2498 (1984)

31. R.J. De Boer, P. Hogeweg: J. Theor. Blol. 120, 331-351 (1986)

32. R.J. De Boer, P. Hogeweg: J. Theor. Blol. 124, 343-369 (1987)

33. I.G. Kevrekidis, A.D. Zecha, A.S. Perelson: In Iheoretical Immunology, ed. by A.S. Perelson, Part One, SFI Studies in the Science of Complexity, Vol. III (Addison-Wesley, 1988) pp. 167-197 